\title{
Improved Predictions for Superconductors
}

\section{A refinement of the density-functional theory used to describe conventional superconductors dramatically increases the accuracy of the theory's predictions.}

\section{By Matteo Rini}

\begin{abstract}
$\Lambda$ holy grail of condensed-matter physics is the development of tools that can predict, from first principles, key properties of superconductors. Such tools could guide the search for new materials that superconduct at higher temperatures. The current computational method of choice is superconducting density-functional theory (SCDFT)-a version of DFT that accounts for many-body effects from electronic correlations in the material. SCDFT, however, systematically underestimates a superconductor's critical temperature $\left(T_{c}\right)$, with dramatic errors for several important materials. Now, Antonio Sanna, of the Max Planck Institute of Microstructure Physics in Germany, and co-workers have corrected this problem by refining the functionals used in SCDFT. Using their revised functionals, they find that the SCDFT's predictions achieve reasonable agreement with experimental data.
\end{abstract}

SCDFT determines the properties of a many-electron system

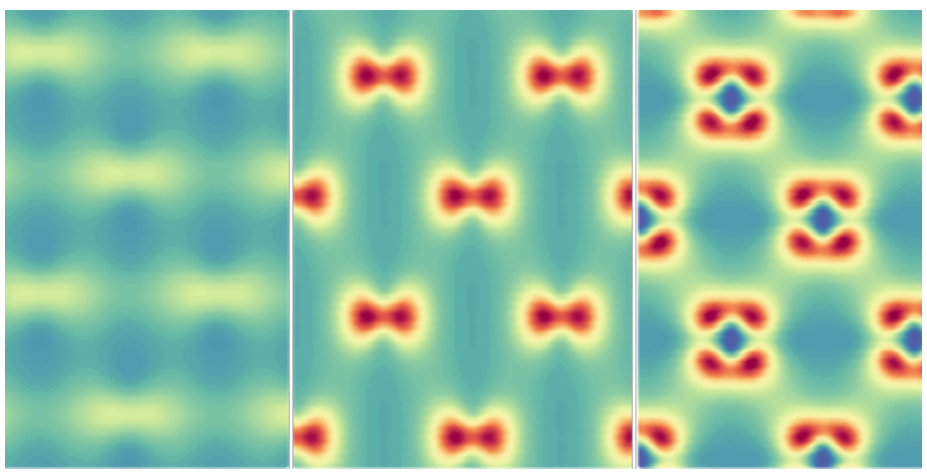

Credit: A. Sanna/MPI for Microstructure Physics; C. Pellegrini/The Hebrew Univ. of Jerusalem using functionals that account for the material's electron density. To make calculations feasible, these functionals neglect certain corrections to the system's energy. But theorists believe that this approximation leads to inaccurate accounting of the electron-phonon coupling that underpins superconductivity.

The team's new approach obtains the functionals without the drawbacks of that approximation. They show that their functionals, which they express with simple analytical formulas, can be used in SCDFT without increasing computational costs. To test their formalism, Sanna and colleagues calculated the superconducting gaps and transition temperatures for a range of superconductors, including elemental compounds, such as aluminum and tantalum, and binary systems, such as magnesium diboride and sulfur hydride. The results show a significant improvement in accuracy compared to previous methods, with calculated $T_{\mathcal{C}}$ 's within $20 \%$ of the real ones for all the studied materials.

This research is published in Physical Review Letters.

Matteo Rini is the Deputy Editor of Physics. 